\title{
LAS POLÍTICAS PÚBLICASY SU PERSPECTIVA DEANÁLISIS: UN CAMPO ABIERTO PARA LA INVESTIGACIÓN Y EL ENTENDIMIENTO DE LOS PROCESOS DE PARTICIPACIÓN
}

\author{
Isabel Cristina Uribe Martínez*
}

\begin{abstract}
Resumen
Este ensayo entonces, es escrito en el marco de la investigación: Políticas públicas y gobierno público en Medellín, estrategias de consolidación del derecho público con perspectiva territorial. Esta Investigación es desarrollada por el grupo de investigación de la Facultad de Derecho de la Universidad Autónoma Latinoamericana de Medellín, que desde el principio ha tenido como propósito fortalecer la línea de investigación de Derecho Público que caracteriza a esta Facultad, mostrando la relación íntima existente entre las políticas públicas y el Derecho público, debido a que una vez se toma la decisión que da origen a la política pública, ésta se materializa, en la mayoría de los casos, a través de normas que entran a jugar parte dentro de lo que se considera como Derecho Público. Estas normas, además, son las que en determinados momentos le asignan a la sociedad la posibilidad de intervenir, así sea indirectamente, en la toma de decisiones por parte del Estado, siendo entonces esta intervención condición sin la cual no se podría hablar de la existencia de dichas políticas.
\end{abstract}

Palabras Claves: Políticas Públicas, gobierno público, actores sociales, públicos organizativos, agenda pública.

\begin{abstract}
This essay, then, is written in the frame of the research: Public policies and public government in Medellín, strategies to consolidate public law with a territorial perspective. This research is carried out by the research group from the School of Law of the Autónoma Latinoamericana University from Medellín, which, from the beginning has had as its purpose the strengthening of the line of research for Public Law, which characterizes this school; showing the intimate relationship existing between public policies and public law since, once a decision is made public policy is born. This materializes, in most of the cases, through rules that play roles in what is considered Public Law.
\end{abstract}

Furthermore, these rules are the ones that empower civil society to intervene, even indirectly, in the state decisionmaking processes, being this intervention a condition sine qua non to talk about said policies.

Key words: Public policies, public government, social actors, organizational audiences, public agenda.

\footnotetext{
*Abogada Universidad Autónoma Latinoamericana. Perteneciente al Grupo de Investigación Ratio Juris de la Facultad de Derecho de Unaula, Coinvestigadora proyecto: POLíTICAS PÚBLICAS Y GOBIERNO PÚBLICO EN MEDELLÍN, ESTRATEGIAS DE CONSOLIDACIÓN DEL DERECHO PÚBLICO CON PERSPECTIVA TERRITORIAL. Email: isaucristina@gmail.com.
} 
nación de la razón en la historia, como el brazo ar-

LAS POLITICAS PÚBLICAS Y SU PERSPECTIVA DE ANÁLISIS: UN CAMPO ABIERTO PARA LAIMVESTIGACIÓN Y EL ENTENDIMIENTO DE LOS PROCESOS DE PARTICIPACIÓN

\section{Introducción}

Durante mucho tiempo el Estado fue considerado como un ente estático, conformado por un sin número de instituciones y órganos que se entrelazaban y creaban una institución investida de poder. Durante este tiempo y debido a la concepción que se tenía de él, el Estado fue abordado por los estudiosos de la Ciencia Política a partir de estas instituciones, respondiendo a interrogantes tales como: ¿Por qué tales instituciones y no otras? ¿De dónde derivan su legitimidad? ¿Por qué se conforma como ese Estado y no como otro?, generando así un concepto de Estado inerte e inmóvil frente a las prerrogativas sociales.

En el Siglo Veinte, como lo plantea Hernández (1999):

El Estado, sin duda alguna es el fenómeno político dominante en el Siglo Veinte, es el producto de la acumulación de estructuras $y$ de prerroen el curso de la historia reciente. Reivindicando progresivamente un espectro cada vez más amplio de funciones sociales, el Estado constituye en torno a sí un aparato burocrático encargado de funciones $d e$ regulación social que devienen gradualmente más complejas. (p.80)

Esto porque poco a poco, y a partir de la segunda guerra mundial, surge un modelo de Estado conocido como el Estado Bienestar en el que el pape del aparato gubernamental es mucho más activo, cambiando la visión de quienes se dedican a su estudio, de un Estado netarnente institucional a un Estado que cumple un sinnúmero de funciones y de actividades para el logro de sus cometidos.

Debido a este cambio, es que se puede afirmar que si antes el Estado era percibido como la encar- mado de la burguesía o como el detentor del monopolio de la violencia legítima, en la actualidad el Estado se concibe a través de su actividad.

Al cambiar la visión que se tenía del Estado, se empieza a pensar en una forma de estudio que transformará los conceptos hasta ahora manejados para su abordaje.

El estudio de la actividad estatal concebida como política pública, surge bajo la dimensión de Análisis de Políticas públicas que se inicia a partir de los años setenta, con un movimiento que se despliega en los Estados Unidos y que luego irrumpe en el continente Europeo. Para los años setenta Alemania ya hacía parte de éste, que ya era considerado un gran movimiento y para los años ochenta, la acogida ya tomaba dimensiones de carácter mundial.

En Latinoamérica según Roth (2006, p.11), “Los investigadores latinoamericanos se dedicaron más bien a estudios clásicos de ciencia administrativa como el análisis de las especificidades de la burocracia estatal en América Latina." Entre estos investigadores Roth destaca a Oscar Oslak y Bernardo Klikberg.

El análisis de políticas públicas, debido a su novedad, fue abordado a partir de variados enfoques. Uno tradicional que consideraba en los años cincuenta y sesenta que las políticas públicas eran variables que dependían de manera directa y necesaria de la actividad política. De esta manera, las políticas públicas sólo eran consecuencia de los gobernantes o de quienes detentaban el poder a través de la representación. Roth (2006).

Al pasar de los años, se empieza a dejar de lado los problemas de representatividad, participación electoral, y se cambia el interés por la manera como se implementan y se elaboran las políticas públicas, y por quienes son elaboradas e implementadas, el análisis de políticas públicas desplaza

\section{RATIO JURIS No. 8}

su interés hacia la actividad concreta del Estado considerándola de una manera independiente de gobernante de turno. Roth (2006).

En este ensayo, se abordarán varias dimensiones dirigidas a responder interrogantes como: ¿Qué es una política pública?, ¿Cuáles son sus características? ¿Cómo se clasifican?, ¿En qué consiste el análisis?, ¿Cuáles son las herramientas para el análisis?, estas son respuestas que permiten tener una comprensión más amplia sobre la importancia de las políticas públicas y cómo el análisis contribuye a su desarrollo e implementabilidad.

En primer lugar, después de esta introducción, se abordará el concepto de políticas públicas a partir de las dimensiones que hasta ahora se conocen de ella, para luego abordar el Análisis de Políticas públicas, los enfoques de análisis y las esferas en que la política pública es analizada mostrando el ciclo de vida de las mismas. Para pasar por último a presentar las conclusiones pertinentes sobre el tema.

\section{Políticas Públicas}

Es necesario elaborar un concepto de algo para poder comprender sus fenómenos. Es por esto que se inicia mostrando los diversos conceptos o acepciones que se tiene sobre políticas públicas partiendo de una tradicional dificultad semántica que señalan la mayoría de los autores relativa a término en español de política, pues bajo esta palabra se entienden tres significados distintos frente a los que no se hace distinción alguna, y que en el idioma inglés sí admite diferenciación.

En primer lugar, política entendida como gobierno de las sociedades humanas, polity en inglés. En segundo lugar, política concebida como la lucha organizada por el control del poder, politics en inglés y en tercer lugar, política como propósitos y programas de las autoridades públicas, es decir política en un plano decisional, policy en inglés. Resta decir entonces, que es a Policy a lo que se hace referencia para hablar de políticas públicas.
Existe un gran número de definiciones de política pública que terminan mostrando la consideración subjetiva que muchos de los autores manejan sobre el tema y la manera como la conciben. Para Salazar (1995, p.30) las políticas públicas son "E conjunto de sucesivas respuestas del Estado frente a situaciones consideradas socialmente problemáticas." Salazar señala que cuando habla de un conjunto de respuestas del Estado, quiere significar que casi nunca una política pública es una sola decisión, sino que por lo general involucra un conjunto o "rosario" de decisiones que se pueden dar de manera simultánea o secuencial en el tiempo. Dice que son del gobierno o del régimen político. En cuánto hace alusión a que son respuestas frente a situaciones consideradas socialmente problemáticas, quiere significar que en cualquier sociedad hay problemas por resolver y que no siempre el Estado logra enfrentarlos por razones que pueden variar desde la falta de recursos, tiempo, presiones, interés; terminando el Estado por enfrentar aquellos que generan más presión o tienen mayor incidencia, situaciones estas que suelen provenir de grupos sociales.

Para Mény y Thoenig (1992, p. 89) "Una política pública es el resultado de la actividad de una autoridad investida de poder público y de legitimidad gubernamental".

Heclo y Wildavsky (1974), en una definición citada por Roth (2006, p.26) exponen una definición simple: “una politica pública es una acción gubernamental dirigida hacia el logro de objetivos fuera de ella misma."

Roth $(2006$, p. 27) define la política pública diciendo:

(...) considero que una política pública designa la existencia de un conjunto conformado por uno o varios objetivos colectivos considerados necesarios o deseables y por medios y acciones que son tratados, por lo menos parcialmente, por una institución u organización gubernamental con la finalidad de orientar el compor- 
tamiento de actores individuales o colectivos de ella, directa o indirectamente, son personas." para modificar una situación percibida con insatisfactoria o problemática"

De esta manera es definida la política pública en autores extranjeros. ¿Pero cómo lo definen los autores colombianos?

Lozano $(2008$, p.16) define la política pública con una óptica que desde ya permite entrever una serie de características que se descubren en autores ya clásicos frente al tema, diciendo que:

Las políticas públicas corresponden a procesos de naturaleza económica, social, política y cultural, caracterizados por formas de intervención de estado encaminadas a solucionar problemas considerados socialmente relevantes, atendiendo a ámbitos de actuación, continuidad y sostenibilidad en el tiempo por parte de autoridades estatales; además de medios de estabilización y coerción que garanticen el logro de objetivos, la generación de resultados esperados, la consecución de condiciones deseadas y de comportamientos sociales admisibles.

Por último, la definición dada por Vargas (1999, p.57), parece distanciarse un poco de las demás en cuanto a su concepción del origen y procedencia de las políticas públicas. Definición en la que se ve enmarcado el trabajo del grupo de investigación de Derecho Público y Políticas Públicas de la Universidad Autónoma Latinoamericana, al decir que una política pública es "el conjunto de sucesivas iniciativas, decisiones y acciones del régimen político frente a situaciones socialmente problemáticas y que buscan la resolución de las mismas o "levarlas a niveles manejables."

En cada una de las definiciones se puede observar la presencia de unos actores que influyen en la política pública, pues según Roth (2006) “(...) hay que tener en cuenta que la acción gubernamental se desarrolla para y a través de actores sociales. Son seres humanos quienes la conciben, la deciden y la implementan, e igualmente los destinatarios (p.27)

Los actores en las políticas públicas son de tipo político y/o de tipo social. Los de tipo político son los partidos y los movimientos políticos. Los actores sociales tienen relación con lo que se considera como movimientos sociales $u$ organizaciones nerar presión en el Estado para que considere un problema colectivo, como un problema de interés general de tipo político y que frente a éste genere respuestas en términos de políticas públicas, dándose un tránsito, de una situación subjetivamente existente, a una situación objetivamente conside rada, precisamente por la intervención de los actores en su definición y evaluación. Salazar (1995)

Las políticas públicas en las que intervienen estos actores, se desenvuelven en dos dimensiones fundamentales que complementan su definición y establecen criterios de definición entre varias políticas. La primera de estas dimensiones, es la temporal, que logra situar la problemática en un momento determinado y permite diferenciar el problema que se presenta en ese momento, con la presentada en un mom dimensión, es la espacial, porque como es lógico las políticas públicas varían de un lugar a otro; diferencia que no sólo se establece por la condiciones de los distintos espacios, sino también porque esas condiciones espaciales generan comportamientos sociales, económicos, políticos y culturales diversos. Mény y Thoenig (1992).

De acuerdo con estas dos dimensiones no será lo mismo una política pública referida a la movilidad en Medellín en el año 1986, a una política pública de movilidad en Medellin para el año del 2008. que tampoco será lo mismo, una política pública de educación para Medellín en el año 2002, y una política pública de educación para Bogotá en el mismo año.

Ya se ha afirmado, que existen elementos que permiten establecer diferencias entre una política $y$ otra, como lo son las dimensiones espacial y temporal. Gracias a la posibilidad de establecer difeporal. Gracias a la posibilidad de establecer diferencias, surge la clasificación de las políticas públi-
cas, que constituye un elemento fundamental al momento de ser analizadas.

La literatura sobre políticas públicas, ofrece una abundante $y$ enriquecedora lista de clasificaciones, entre ella y tal como lo hace Thoenig (1997) podríamos citar aquellas que se construyen sobre los instrumentos de influencia utilizados por el Estado (Scharp, 1976), sobre los instrumentos administrativos (Mayntz, 1976) o sobre la forma de intervención sociopolítica (Kauffmann, 1982).

Como se dijo anteriormente, estas clasificaciones ofrecen un elemento puntual para el análisis de políticas públicas por lo cual al momento de elegir una clasificación determinada según Thoening (1997) debe hacerse en función de dos consideraciones: primero, la pertinencia de los criterios, con relación a la finalidad de lo que se desea analizar. Segundo, la cualidad de la reflexión de los conceptos y teorías sobre los conceptos.

Hay una clasificación que es frecuentemente citada por los autores que tratan sobre este tema, y es la elaborada por Lowi (1972):

"Políticas públicas distributivas": son aquellas que autorizan $o$ conceden un privilegio.

"Políticas públicas redistributivas": son aquellas que clasifican a las personas según ciertos criterios que les dan o no acceso a ciertas ventajas.

"Políticas públicas constitutivas": son aquellas que definen medidas sobre las leyes y el poder.

"Políticas públicas reglamentarias": que se divide en dos categorías, unas que limitan intereses y la libertad de las personas a las que se aplican, las otras, conocidas por algunos como autorreglamentarias, son aquellas que aumentan los privilegios del público al cual se dirige.
Esta clasificación permite ver cómo las políticas públicas tienen un elemento teleológico, que persigue producir efectos. Estos efectos pueden ser previstos al momento de diseñar una política pública haciendo un intento por reducir al máximo la incertidumbre, muchos de los elementos para a previsión de efectos lo da el análisis de las políticas, pero a veces surgen efectos que de ningún modo se hubieran podido prever, y que afectan de la misma manera tanto al grupo o a los grupos determinados de la política, como a otros grupos que no tenían nada que ver con ella. Estos grupos, son aquellos que están en la misma línea de acción y sufren las consecuencias, ya sean positivas o negativas. Thoenig et al. (1992).

Los elementos vistos hasta ahora, partiendo de la definición de políticas públicas permiten avanzar con paso firme a lo que se considera el centro de este trabajo: El análisis de las políticas públicas, que permite una aprehensión más amplia sobre este "nuevo elemento" que transforma el objeto de estudio de la ciencia política y del derecho público, contribuyendo a que sus profesionales vislumbren nuevos campos de acción en torno a la investigación.

\section{Análisis de políticas públicas}

Este capítulo será abordado a partir de la concepción que se tiene del análisis de políticas públicas, frente a la cual algunos plantean que es una disciplina, mientras otro opinan que se trata sólo de otra forma de estudiar y conocer la actividad del Estado desde su fase decisional.

El análisis de políticas públicas surge a partir de cambio del Estado, de un Estado estático a un Estado dinámico e intervencionista a partir del Siglo Veinte, situación que reclama una nueva forma de análisis del Estado a partir de la actividad que despliega para el cumplimiento de su función.

Este análisis debido a su carácter práctico y a la vez teórico, ha hecho que se vea atravesado por 
corrientes heterogéneas, determinadas en la mayoría de las veces por las problemáticas políticas dominantes del momento, tal y como lo expresa Roth (2006):

De las dificultades del modelo del Estado de bienestar en los años sesenta y setenta (déficit de resultados), pasaron a su crítica en la década de los años ochenta (corporativismo, burocratización) y a la respuesta neoliberal en los años noventa (privatizaciones). Los analistas de los años setenta se enfocaron particularmente sobre el tema de la implementación de las decisiones. En los años setenta el tema principal fue el análisis comparativo de los modelos de Estado providencia; y luego, en los años ochenta, el centro de interés se desplazó hacia la problemática de la constitución de las agendas gubernamentales y de las configuraciones (sistemas, redes, comunidades) de actores que influyen en las políticas públicas. Finalmente, en los años noventa, con la ola neoliberal, muchos analistas preocupados por la eficiencia de la gestión pública debatieron alrededor de la reforma administrativa con la famosa nueva gestión pública y se incrementó el interés por evaluación de la acción pública. (p.14)

En el intento por definir el análisis de políticas públicas, la mayoría hace énfasis en que las políticas públicas por su cercanía a la acción estatal logran la solución de una serie de problemas en relación con la función que cumple el Estado en la sociedad.

En la definición dada por Dunn (1981) citado por Thoening (1997, p. 65), se rescatan dos características que podrían mostrar la importancia del análisis para la solución de problemas y la efectividad en la toma de decisiones; estas características serían primero, el carácter pluridisciplinario del método científico y segundo la orientación de éste hacia e apoyo de la decisión:

El análisis de políticas públicas es una disciplina de las ciencias sociales que utiliza múltiples métodos de investigación y de argumentación para producir y transformar la información pertinente de ciertas políticas, información que puede ser empleada dentro de los contextos políticos a fin de resolver los problemas de la política acción.

Dye citado por Roth (2006), señala el análisis de las políticas públicas como algo que:

(...) tiene que ver más con la explicación que con la prescripción, pues consiste en una indagación rigurosa de las causas y las consecuencias de las políticas públicas y que, finalmente, se esfuerza por desarrollar y probar hipótesis generale sobre las causas y las consecuencias de las políticas públicas por medio de la acumulación de investigaciones empíricas de relevancia general (p. 15).

Estas definiciones se consideran suficientes para lustrar al lector sobre el concepto de políticas públicas. Ahora, es básico mostrar la importancia que tiene este análisis y su repercusión en la sociedad , mayormente, en el Estado.

Las sociedades por el paso del tiempo van sufriendo una serie de transformaciones que exigen que el Estado vaya avanzando a su mismo paso o unos pasos más adelante para poder suplir las necesidades. La sociedad actual se encuentra en presencia de una serie de cambios de corte económico, social, político y cultural dentro de un mundo globalizado que exige del Estado una transformación que le permita adaptarse a la necesidad de regulación y de asistencia que dichos cambios implican.

Frente a esta necesidad, el análisis de políticas públicas ofrece una renovación de los estudios para la comprensión del Estado y de sus acciones, que permiten tener información fidedigna de cual es e qué debe consistir su transformación. Es así entonces, como un estudio minucioso de las actividades estatales, en varios temas, ha sido realizado en los Estados Unidos y en Europa. Estos estudios, en la mayoría de los casos, han mostrado la distancia ocasiones importante, entre las intenciones iniciales del Estado o del legislador y las realizaciones concretas. De esta manera el análisis de las políticas públicas ha contribuido significativamente a la comprensión del Estado como un sistema de acción específico con vocación de coordinar los otros ción específico con
sistemas de acción.

El análisis de políticas públicas, permite, sin deci que el Estado lo es, llevar a que éste sea mirado como una organización común a través de la cual los agentes públicos persiguen unos intereses que no se constituyen de manera exclusiva en la respuesta esperada por los actores sociales a parti de sus reivindicaciones, pero a la vez, el Estado pue sus revindicaciones, pero a la vez, el Estado puede ser concebido como una organización capaz de influir en todos los ámbitos que se presentan en la sociedad. Thoening et al. (1992)

La importancia entonces, del análisis de las políticas públicas, está en la posibilidad de transformar aquello que analiza ya sea la decisión del Estado te identificar cuales son los vacíos que el Estado no a icanza a llenar a través de la expedición de leyes, alcanza all lonar a traves de la expedición de leves, del uso del derecho en general y de su institucionalidad. De esta forma, el análisis permite establece cuál es el nivel de influencia que tiene la sociedad como organización social en la toma de decisiones cubernamentales, $y$ como este transforma situacubernamentales, y como este transforma situanciben como asuntos colectivos, para pasar a convertirse en asuntos de interés general, en otras palabras, en asuntos políticos. El análisis de políticas públicas, también permite prever cuáles son los efectos cas, tambien permite prevercúa a partir de su imresultados que se producirán a partir de su implementación, fortaleciendo de esta forma la toma de decisiones por parte del Estado, que es un proceso selectivo y limitado, donde el número de actores es reducido, lo que permite revelar entonces to forma como está repartido el poder dentro de Estado, concluyendo como lo hace Roth (2006, p. 20) "De manera que a través del análisis de esto procesos políticos, que legitiman y concretizan la orientación de las políticas públicas, es posible dar cuenta empíricamente del tipo de Estado, de su evolución y transformación.

Como ya se ha podido observar, el análisis de las políticas públicas, no es una nueva teoría del Estado, sino que es una nueva forma de observación, o como ya se ha dicho, es una nueva forma de escomo ya se ha dichor enfoques como lo son aquellos que se centran en la sociedad, los que se centran en el Estado y e enfoque mixto o intermedio. Enfoques todos ellos que reven una forma específica que se ve reflejada en el resultado del análisis.

El enfoque que centra su atención en la sociedad comprende el Estado como un organismo que depende de ella. Según Roth (2006 p. 29) "Es el repultado del estado de las relaciones de fuerzas entre, según unos, las diferentes clases, fracciones de clases o naciones, y según otros, entre individuos y grupos que determina la elección de las políticas desarrolladas por la institución estatal." En este destro enfoques marxistas y neomarxistas de la sociedad.

El enfoque centrado en el Estado, es todo lo contrario anterior consideran al Estado como un ortranismo independiente a la sociedad, quien es el encargado de recibir las demandas, seleccionarlas $y$ proveer servicios. Es el Estado entonces el mayor decisor en materia de elección de las políticas públicas. Este enfoque tiende a disminuir la inpúbibles en a sociedad para explicar las elecciones de políticas públicas. Roth (2006)

El enfoque mixto, como su mismo nombre lo indica e ubica un unción media rechazando tanto se ublca en una posicion media, la sociedad y la idea de un Estado que minimiza la participación de la sociedad en la toma de las decisiones. Thoening et al. (1992)

importancia de hacer mención de estos tipos de La importancia de hosibilidad de afirmar, como 
lo hace Roth, que a pesar de que el análisis de las políticas públicas es posible en todos los enfoques, es en el mixto en donde se percibe la mayor posibilidad de generar unos resultados con posiciones más abiertas, menos radicales, que contribuyan a enriquecimiento de esta nueva forma de conocer y observar el Estado en relación con la sociedad mucho más cuando es este enfoque el que permi-
te hablar del análisis en red, que es definido como te hablar del análisis en red, que es definido como tura conformada por un conjunto de actores, donde la colectividad es más importante que la misma individualidad, lo que genera una serie de características que enriquecen la labor analítica.

Otro de los enfoques de análisis de las políticas públicas que hace parte de los elementos que Thoening ha denominado caja de herramientas (1992). Es EL POLICY CYCLE, que se convirtió en una de las formas más pedagógicas para comprender las etapas de análisis de políticas públicas, por ofrecer una serie de secuencias lógicas, que en un primer momento parecieran tener un orden lineal incorruptible, pero que según Thoening (1997), estas etapas lógicamente desarrolladas, pueden incluso saltarse la una a la otra, incluso no darse una o varias de ellas, o la que es considerada la última podría convertirse en la primera y viceversa. En palabras de Thoening:

(...) no se hace evidente que otra fase siga $o$ anteceda necesariamente a la anterior: las fases podrían llegar a superponerse, invertirse en su secuencia cronológica o, aún en ciertas actividades funcionales, pueden inclusive no aparece en el proceso. De acuerdo con lo anterior, para que las políticas públicas finalicen, no resulta necesario que las actividades de evaluación se realicen: la política pública puede desaparecer porque nunca se puso en marcha, o se puede perseguir indefinidamente sin que nadie piense jamás en asimilarla con los resultados" (p.80).

Es así, como esta propuesta elaborada por Jones (1970) hace énfasis en cinco esferas en la vida o desarrollo de una política pública: primero, iden- tificación de un problema, segundo, formulación de soluciones, tercero, toma de decisión, cuarto, Implementación y quinto, Evaluación.

Este modelo por su generalidad, permite la utilización con cualquier clase de política pública, permitiendo una identificación clara de los escenarios, de los actores, generando así una delimitación específica del objeto de estudio.

En la primera esfera, una determinada situación es percibida como un problema por los actores, $y$ a sean de tipo político o social como se decía anteriormente. Los actores entonces, traducen el problema solicitando una acción pública que permita convertir su problema en un problema de interés general de tipo político para ser insertado en la agenda del sistema gubernamental. En la segunda esfera, una vez el asunto, como lo denomina Salazar (1995), es logrado incluir en la agenda gubernamental, la administración trata de buscar soluciones posibles. En la tercera esfera, los actores autorizados para tomar las decisiones, se recuerda que es un grupo limitado y selectivo que depende de la distribución del poder dentro del Estado, para tomar la decisión analizan las soluciones $y$ respuestas posibles y deciden la que ellos consideran más apropiada de acuerdo a una serie de factores como los económicos, sociales, culturales, políticos, ambientales o de juego de intereses, entre otros. Se debe recordar además, que según Thoenig (1992) y varios autores, sostienen que la no decisión también constituye una forma de política pública. La cuarta esfera, tiene relación con la implementación, también es posible su no implementación. La quinta esfera, que no se analizará en este trabajo por considerar que su estudio es específico y requiere mayor rigurosidad, es la evaluación, que por lo general es realizada por los actores sociales que promueven la iniciativa y por los actores de tipo político. La evaluación entonces, puede dar lugar a un reinicio de todo el ciclo con la finalidad de reajustar la respuesta, método que algunos llaman retrospectivo y que puede dar lugar a la eliminación de la política. Roth (2006).
A pesar del aporte pedagógico en el que se conA perte de aporte pula cas que llevan a privilegiar el tercer enfoque visto sobre la teoría mixta y el desarrollo del análisis en redes que se propone a partir de éste.

Sabatier (1988 p. 129) citado por Roth (2006 p.52), crítica este modelo por:

(...) su incapacidad de dar explicaciones de carácter lógico por medio de la formulación y comprobación de hipótesis. No permite responder a preguntas de tipo: ¿cuáles son las condiciones que transforman el proceso de una fase a otra? Otra limitación, es la tendencia a situar la evaluación sólo al final del proceso.

Según Sabatier, el proceso de evaluación es hecho por los actores de manera formal o informal en todo lo largo del proceso para evitar que se presenten errores $y$ en caso de que estos se presenten, entrar a corregirlos a tiempo antes que sea demasiado tarde. Este mismo autor, plantea que es un enfoque que privilegia sobremanera la institucionalidad y el legalismo, que no sería la forma apropiada para acercarse al conocimiento de las apropiada para acercarse al conocimiento de las de hacer un recorte en fases o etapas, dificulta mostrar las conexiones existentes entre ellas y la reiteración entre los ciclos, que en algún punto generarían una visión sesgada del proceso de la política pública como tal.

Otros autores, por su parte, centran sus críticas en la dificultad que se tiene para la comprensión y separación d tación y la evaluación.

El policy cycle postula que la implementación es una etapa postdecisional, y que la administración encargada ejecuta la política según los textos escritos; por lo tanto, si se presentan problemas durante la implementación se trata de un problema técnico que se puede mejorar. Roth (2006, p. 52)
Muller y Surel (1998) citado por Roth (2006) critican este enfoque por ser un modelo que tiende a hacer creer que las políticas públicas sólo sirven para dar solución a los problemas que se le prepara dar solución a los problemas que se le presentan a la administración para su decisión. Por cas públicas no solucionan problemas, sino que los problemas son solucionados por los actores sociales al implementar estrategias y acciones frente a un problema que le es común a un sector determinado de la sociedad.

A pesar de las críticas hechas a este enfoque, resulta ser este la mejor manera de acercarse al conocimiento del proceso de elaboración de una política pública para efectos de comprender en qué consiste su análisis, pues cada una de las etapas propuestas por Jones (1970) permite una estructura de carácter pedagógico, que es la propuesta que se extiende al lector. Por esta razón se pasará a desarrollar cada una de las etapas.

\section{Identificación de un problema}

¿En qué punto un determinado problema, se convierte en objeto de acción para un ente administrativo o Estatal?

En el punto en que este problema logra ser configurado como un asunto de interés general, público o político que merezca un lugar en la agenda de gobierno para ser discutido públicamente por el decisor. Padioleau (1982), citado por Thoenig (1992 p. 126) define la agenda como "el conjunto de problemas percibidos cuando se hace un llamamiento a debate público, incluso cuando existe intervención (activa) por parte de las autoridades públicas legítimas" Esta definición de agenda es la más utilizada por los autores.

La incursión de un problema en la agenda puede presentar diversos grados de consenso o conflicto. Según Thoening (1992), existen varias situaciones, por un lado están las situaciones en las que el acceso se debe a que es algo fijo, casi como una rutina 
dentro de la agenda de gobierno, ejemplo de esto es la discusión del presupuesto que se hace cada año, frente a temas como estos, el debate se hace de una manera codificada y regido por los formalismos establecidos para el caso particular, incluso en la Constitución. Del otro lado, el acceso de un problema a la agenda gubernamental, está marcado por la controversia, se presentan conflictos incluso con otros problemas que también ameritan su inclusión en agenda para debate por lo cual se deben establecer prioridades.

Como se logra ver, el acceso de un problema o asunto a la agenda gubernamental, sin importar cual sea, siempre tiene límites. Estos límites están marcados por las condiciones que se deben cumplir para poder entrar en agenda. Se debe analizar si el problema es de competencia de una autoridad pública determinada. El hecho de que se tenga que analizar la competencia, no implica que vaya a existir de manera determinante una acción de la autoridad pública competente para resolver el problema. Éste se inserta, lo único que queda es la esperanza de que se obtenga el resultado esperado. De acuerdo con Thoenig (1997), lo que se puede presentar, es que gracias a las intervenciones de los elegidos a favor de sus electores cuando de servicios del Estado se trata, lo importante es mostrar que se está atendiendo a sus solicitude de manera que el resultado real, sea prácticamente secundario. Resultan siendo estos problemas después de ser insertados en la agenda, como ofertas a la espera de que al menos sean debatidos públicamente y que no se echen al olvido.

Otra condición, es que el problema logre ser traducido o traducible a la lengua de la acción pública. Thoening $(1997$, p. 85) plantea que:

(...) para que un problema social se convierta en el problema político, se necesita plantea los términos que estén en juego para el ámbito político oficial, puesto que siempre habrá intereses que se estén arriesgando con cada discusión, gracias a la dinámica de la marcha misma del proceso.

Como última condición es necesario que el asunto sea considerado como una situación problemática dentro o fuera del sistema político, lo que hace entonces el analista de políticas públicas es observar la diferencia entre las relaciones, los hechos percibidos, las aspiraciones y los hechos tal cual serán vistos por quienes perciben finalmente la situación. Thoenig (1997).

Que un problema se inserte en la agenda, es producto de la inestabilidad y del conflicto, es por esto que el analista debe actuar prudentemente al momento de establecer objetivos o puntos de análisis, pues "La puesta en la agenda es un trayecto lleno de representaciones, sentidos y significados propios a los actores concernientes."Thoenig (1997, p. 85) El analista además de ser prudente, debe buscar la manera de definir cuáles son las estrategias y maniobras de las autoridades que tienen la labor de debatir los asuntos frente a sus opositores y a los diversos tipos de públicos, ya que el proceso $y$ su dinámica terminan por ajustarse tanto al problema, como a sus probabilidades de acceso a la agenda.

Cobb y Elder (1976) citado por Thoenig et al. (1992) hacen especial énfasis en el papel que juegan los medios de comunicación al momento de incluir un asunto en agenda, quienes tienen la habilidad de acelerar o frenar las actividades.

El analista por último, debe saber entender cuales son las formas de expresión y de conciliación en medio del conflicto que se pueden presentar por la presencia de actores ya sean de tipo social o potico que generan grupos de presión, pues esto le permitirá tener claro su influencia al momento de inserción de un asunto en la agenda de gobierno.

\section{Formulación de Soluciones}

Una vez el problema logra ser insertado en la agenda de gobierno $o$ agenda política como la define Roth (2006), se pasa a la formulación de soluciones. En este proceso se selecciona cuál de las posibles alternativas disminuyen la tensión entre el ser y el deber ser, resultando ser la solución más apropiada o factible.

Para esto, según Roth (2006) se dispone de varias técnicas metodológicas que hacen más fácil la formulación de una solución. Según el Cetel (2000) citado por Roth (2006) en primer lugar resulta importante y básico tener claridad sobre los fines y metas de la política a diseñar, para luego precisar los objetivos concretos. La meta lo que logra es establecer la dirección a seguir. El objetivo determina lo que se espera en caso de producirse la política pública.

Si el analista logra determinar cuáles son los fines $y$ los objetivos que se tienen determinando una especie de cascada donde se demarque la jerarquización, podrá establecer cuál va a ser el nivel de intervención del Estado y la justificación de las medidas que se tomen. Generalmente, o mejor dicho debe ser así, los fines o metas que se esperan en una política pública en especial, están trazados en la Constitución y la ley como referente de legalidad para la toma de una decisión. Roth (2006).

Un segundo momento, en la formulación de soluciones consiste en hacer una ponderación de lo distintos objetivos que pretenden facilitar la realización de un fin especifico. Roth (2006).

Por último, luego de haber hecho la jerarquizació entre fines y objetivos, y haberlos ponderado, se debe determinar cuáles son los efectos esperados $y$ los indicadores que permitan dar cuenta del grado de realización de la meta. Roth (2006).

Toma de decisión

Una vez analizadas las metas y los objetivos que se alcanzarían con una determinada solución, quien es encargado de decidir toma la decisión. Esta decisión según los diversos enfoques, es tenida, como lo plantea Thoening (1992, pág. 88)
La decisión resulta de un cálculo con miras a escoger, teniendo en cuenta el objetivo que ha de ser alcanzado, la mejor solución, es decir, aquella que sea la más adaptable, la más razonable. Es la inteligencia del decisor lo que importa, junto con la calidad de los criterios técnicos de selección.

En otros enfoques, el proceso de decisión se describe así:

La alternativa que se ha escogido se impone, puesto que ésta es el resultado de un cierto número de procedimientos a través de los cuales pasa la elaboración. Estos procedimientos son rutinas organizacionales o burocráticas, modos de división de funciones entre las unidades 0 circuitos de información que participan en la elaboración. Éstas imponen su lógica propia de funcionamiento, a la solución que es finalmente escogida. El decisor resulta ser, entonces, apenas un prisionero del sistema: son el sistema, sus reglas y sus rigurosidades los que toman finalmente la decisión. Thoening (1992, pág. 89).

Por último, la decisión como producto del poder: (...) la decisión refleja el resultado de un proceso de poder y de influencia, de negociaciones $y$ de conflictos, entre los diferentes puntos de vista que se presentan e intervienen durante su elaboración. El decisor ratifica un compromiso que se le impone y emerge de un campo de relaciones de fuerza entre los diferentes actores: a lo sumo, puede realizar algunas alteraciones a nivel de detalles o evitar errores demasiado graves, teniendo en cuenta el débil margen de libertad del cual dispone en realidad. Thoenig (1996, pág. 90).

Frente a esto el análisis de políticas públicas se encuentra enfrentado a una exigencia de aprovechamiento de las actividades. El analista, puede adoptar entre dos maneras de intervención. La primera es atacar el asunto en un todo a partir de la toma de decisión, teniendo en cuenta las situaciones, 
la estructura, la estrategia, los actores, que parezcan haber intervenido ejerciendo presión sobre el camino recorrido hasta la producción del resultado. La otra, es intervenir estratégicamente en la reconstrucción de los momentos más decisivos y más significativos para la toma de la decisión. En este último caso, lo que se presentaría al tomar el proceso por momentos, sería una construcción de límites al proceso de análisis como tal. Thoenig (1996).

\section{Implementación}

Cuando se toma la decisión, el camino a seguir es la implementación de la política pública, como ya se ha dicho, es posible que la política no se implemente debido a circunstancias de orden político 0 económico.

Para muchos autores, la implementación es un asunto que no presenta mayor inconveniente, pues para ellos la implementación es netamente administrativa, con participación de elementos técnicos que ayudan a la ejecución. Un abogado podría pensar, que la implementación se agota con la expedición de una norma que ordena su puesta en marcha, lo mismo sucedería con un decreto, un acuerdo o una ordenanza.

La implementación para el análisis de las políticas públicas, es como lo dice Roth (2006, p. 107) "Ia continuación de la lucha política, con otros medios y otros escenarios."

Roth (2006) plantea que el estudio de las políticas públicas en su mayor parte se generó al observar que a pesar de que se toma una decisión y esta era implementada, el problema que le había dado origen a esta perduraba, en ocasiones, disminuyéndose parcialmente y en ocasiones aumentándose. De ahí que el analista de políticas públicas debe hacer un estudio minucioso de la brecha existente entre la implementación y la solución de las necesidades

reales para la cual se supone fue implementada. El analista no debe perder de vista, que la política pública es formulada, decidida e implementada por personas, y que a su vez son personas las que reciben el impacto, positivo o negativo que estas pueden tener. Además se debe tener en cuenta que los actores de la política pública, especialmente los decisores en la mayoría de los casos guardan un interés particular que desdibuja ese concepto de objetividad, de neutralidad que se supone deben tener y que en últimas sólo se convierte en un ideal que al ser asi, trae serias consecuencias en proceso de implementación.

\section{La evaluación}

La evaluación aunque hace parte del análisis de políticas públicas, ha tomado dimensiones de disciplina de análisis, gracias al interés que ha despertado en muchos estudiosos del tema. Por lo cual el estudio de la Evaluación amerita una discusión diferente, sin dejar pasar de lado ninguna de sus características. Debido a esto, autores como Thoenig y Álvarez que tratan el análisis de políticas públicas, al estudiar las etapas del Policy Cycle sólo llegan a la implementación.

En este ensayo, sólo se hará mención de ella para concluir las etapas que plantea Jones con fines pedagógicos, que es la intención de este trabajo, con fundamento en lo presentado por Roth en su obra sobre políticas públicas.

La evaluación, es la encargada de definir si la política pública fue exitosa o no. Parafraseando a Majone, la evaluación es la que se ocupa de recolectar, verificar e interpretar todo lo relacionado con la ejecución y la eficacia de las políticas y programas públicos.

La evaluación entonces, parece ser el punto final en el análisis, pero no se debe descuidar que es posible que ésta esté presente en todas y en cada una de las etapas que conforman el Policy Cycle.

\section{Aportes}

Al mirar la forma como cada uno de los autores concibe el estudio de las políticas públicas, se puede concluir que no hay una definición clara en lo relativo a si el estudio de las políticas públicas es una disciplina independiente o si por el contrario está intimamente relacionada con estudios relativos a la teoría del Estado, la ciencia política, la teoría administrativa y el derecho público. En Colombia particularmente, el tema de las políticas públicas es abordado multidisciplinarmente con relación al Derecho público, al Derecho Administrativo y la Ciencia Política. Esto incluso se ve fuertemente reflejado en los planes de estudio de las Universidades, en los que las políticas públicas sólo son un componente más dentro de los programas de Ciencias Políticas y Derecho.

Partiendo de la lectura de las definiciones sobre lo que los autores consideran como políticas públicas, podría decirse que en su mayoría la conciben como la actividad que despliega el Estado en l cumplimiento de su función. Concepción que no es del todo cierta, pues a partir de investigaciones como las realizadas por el grupo de investigación de Derecho Público y Políticas Públicas de la Unaula, se debe hacer diferencia entre lo que son políticas públicas y políticas de gobierno. Si bien escierto, una política pública surge con la decisión es cierto, una politica pública suge con la decisión que toma el gobierno, quienes logran que se tome esa decisión a partir de la formulación del problema y su fijación en agenda gubernamental, son los actores sociales, a partir de un tipo específico de público, denominado públicos organizativos (orgapúblico, denominado públicosorganizativos (organizaciones sociales, gremiales, grupos de vecinos, sindicatos). Una política de gobierno entonces, es aquella que es formulada por el mismo gobierno para ser discutida en debate público y que hace parte del cumplimiento de su función como autoridad política o gub ridad politica o gubernamental. Es por esto que se concluye que no todas las decisiones que tom el Estado, se traducen en políticas públicas tal y como lo tratan la mayoría de los autores que tocan este tema.
Podría decirse también, que si las políticas públicas, dada su importancia, son el cuerpo, el análisis de las políticas públicas son la vida. Pues a través de las polticas públicas son la vida. Pues a través Cer desnudadas en todo su esplendor, permitiendo una aproximación más cercana a su conocimiento. Al posibilitarse su conocimiento, mucho más factible será su aplicabilidad y la importancia de su estudio. No comprensión de la forma como se relaciona el Estado con la sociedad (análisis de redes), sino porque a partir del análisis de las políticas públicas se tiene la posibilidad de realizar investigaciones que den cuenta sobre lo que está ocurriendo en los municipios, en los departamentos y en el país con el tema de las políticas públicas y del cómo se manejan éstas en las agendas de los diversos actores. El análisis con su caja de herramientas, se convierte entonces, en la vida de la investigación que plantea el grupo de investigación de derecho público y políticas públicas dentro del territorio local, mirando la interrelación de las agendas públicas de gobierno público y agendas gubernamentales de gobierno institucional para la toma de decisiones.

El análisis permitirá establecer, en principio y como primer acercamiento con fundamento el Policy Cycle qué problemas se han concebido en Medellín y cómo los públicos organizativos han logrado su inclusión en agenda. Cuántos de estos se encuentran incluidos, cuáles ya son tenidos como políticas públicas, cuál ha sido o será su implementación, cómo se cumple la evaluación en ellos y cuál es el papel que juega el gobierno público en la ciudad de Medellín.

Sin olvidar que el análisis de todos estos elementos permitirá continuar consolidando el papel que juega el Derecho Público en el gran escenario que ofrecen las políticas públicas, donde cada uno de ellos, se convierte en las esferas en torno a las cuales gira dicha investigación, que para el caso-y vale la pena repetirlo-, se tíula: Polícas Públivalga la pena repetirlo-, se titula: Políticas Públicas y Gobierno Público en Medellín, estrategias 
de consolidación del Derecho Público con perspectiva territorial.

Por último decir, que aunque el tema de las políticas públicas no es tan nuevo para el mundo, en Colombia apenas comienza su auge. El análisis aún es incipiente y esto se ha demostrado en la gran dificultad de encontrar textos especializados para el estudio del tema, por lo general sólo artículos de revistas. Algunos le asignan la responsabilidad a la poca importancia dada a éste por las universidades colombianas, pero valga la pena decir que ya hay algunas que han dado algunos pasos y otras que dan sus primeros pinitos. Muy seguramente Colombia se encuentra en un momento de siembra para luego pasar a recoger buenos frutos, que no se verán sólo desde el punto de vista teórico, sino que se verán reflejados en la realidad.

\section{BIBLIOGRAFÍA}

Hernández, G. (1999). El análisis de políticas públicas: una disciplina incipiente en Colombia. Revista de estudios sociales, 4, 80-91.

Jones, Ch. O. (1970). Una introducción al estudio de las políticas públicas. Barcelona: Ariel.

Lowi, T. J. (1972). Cuatro sistemas de políticas públicas. Barcelona: Ariel.

Lozano, A. A. (2008). Aspectos sobre política pública ejercicios e instrumentos para el análisis. Bogotá: Universidad Nacional de Colombia.

Mény, I. y Thoenig, J. C. (1992). Las políticas públicas. Barcelona: Ariel S.A.

Roth, D.A.. (2006). Políticas públicas. Formulación, implementación y evaluación. Bogotá: Ediciones Aurora.

Salazar, V. C. (1995). Las políticas públicas. Santafé de Bogotá: Pontificia Universidad Javeriana.

Thoenig, J. C. (1997). El análisis de políticas públicas. Universitas, 93, 61-107.

Vargas, V. A. (1999). Notas sobre el Estado y las políticas públicas. Bogotá: Almudena Editores. 